\title{
Clinical confirmation of the American Academy of Neurology algorithm for HIV- 1-associated cognitive/motor disorder
}

\author{
The Dana Consortium on Therapy for HIV Dementia and Related Cognitive Disorders
}

\begin{abstract}
Article abstract-Objective: Our goal was to put into operation the American Academy of Neurology (AAN) algorithm for the definition of minor cognitive/motor disorder and human immunodeficiency virus type 1 (HIV-1)-associated dementia complex (ADC) and examine the neuropsychological, neurologic, psychiatric, and functional deficits in affected subjects. Design: Two hundred seventy-one HIV-positive men and women with CD4 count of $<200$ or demonstrated cognitive impairment were recruited from three sites (Columbia University, The Johns Hopkins University, and the University of Rochester) and underwent standardized assessments. Results: Sixty-five subjects met criteria for ADC (cognitive, functional, and neurologic or behavioral), 56 met criteria for minor cognitive/motor disorder, and 150 met criteria for neither. Seventy-eight subjects met neuropsychological and neurologic/behavioral criteria but did not demonstrate functional impairment. Those with ADC performed significantly worse on speeded motor and verbal memory tests and demonstrated more extrapyramidal signs and behavioral symptoms than did the other two groups. Both ADC and minor cognitive/motor disorder were independently predictive of poor physical function, after adjustment for age, gender, years of education, log (CD4 count), hemoglobin, number of HIV diagnoses and medications, and depression. Conclusions: The operationalization of AAN criteria demonstrates that it is rare to have both cognitive and functional impairment without associated neurologic and/or behavioral deficits. Functional impairment in isolation is also rare. Dementia is an independent predictor of physical function.
\end{abstract}

NEUROLOGY 1996;47:1247-1253

Human immunodeficiency virus type 1 (HIV-1)associated dementia complex (ADC) affects $16 \%$ of patients with acquired immune deficiency syndrome (AIDS) and has an annual incidence of $7 \%$ among persons with AIDS. ${ }^{1}$ In January 1994, the Charles A. Dana Foundation established a consortium of three centers (Columbia University, The Johns Hopkins University, and the University of Rochester) to recruit a cohort of individuals at high risk for the development of $\mathrm{ADC}$ and to conduct a series of phase I/II clinical trials to assess the safety of interventions intended to retard or reverse HIV-related cognitive impairment. The purpose of this report is to describe (1) the characteristics of the cohort; (2) the operationalization of the algorithm for the definition of HIV-1-associated dementia complex and HIV-1associated minor cognitive/motor disorder proposed by the American Academy of Neurology (AAN) AIDS Task Force ${ }^{2}$; and (3) the neurologic, cognitive, psychiatric, and functional deficits in HIV-positive subjects at risk for HIV-1-associated dementia.

Methods. Subjects. To establish a "natural history cohort" for longitudinal follow-up from which participants in the experimental protocols could be drawn, each site planned enrollment of 75 subjects with advanced HIV infection, who are at high risk for the development of $\mathrm{ADC}$, through the use of uniform inclusion and exclusion criteria. Inclusion criteria were (1) being seropositive for HIV based on self-report and confirmed by ELISA and Western blot, (2) being capable of providing informed consent, (3) being ambulatory at the initial visit, and (4) either meeting Centers for Disease Control and Prevention criteria for AIDS (CD4 count of $<200$ ) or demonstrating cognitive impairment (based on the paradigm described below) with a CD4 count of $<300$. Exclusion criteria were (1) current or past opportunistic CNS infection at study entry, with the exception of treated neurosyphilis; (2) history or current clinical evidence of schizophrenia; and (3) history of chronic neurologic disorders such as multiple sclerosis or uncontrolled epilepsy. Current alcohol or drug use was discouraged but was not grounds for exclusion.

Historical information. At entry, a structured questionnaire was administered to each subject to obtain demographic information and medical history, including HIV-1associated illnesses and medications.

Neurologic examination. The neurologic examination was designed to capture signs associated with ADC. The macroneurologic examination created for the AIDS Clinical Trials Group (ACTG) and the motor portion of the Unified Parkinson's Disease Rating Scale (UPDRS) ${ }^{3}$ were performed.

Neuropsychological examination. The neuropsychological battery was designed to delineate ADC and minor cognitive/motor disorder. Where possible, tests recom-

Supported by the Charles A. Dana Foundation and the following National Institutes of Health grants: RR00645 (Columbia University), RR00722, NS26643, AI35042 (The Johns Hopkins University), and RR00044 (University of Rochester).

Address correspondence and reprint requests to Dr. K. Marder, Gertrude H. Sergievsky Center, Columbia University, College of Physicians and Surgeons, 630 W. 168th St., New York, 10032.

Received March 4, 1996. Accepted in final form April 29, 1996. 
mended by the National Institute of Mental Health ${ }^{4}$ and the ACTG were included. Scores for subjects with $\leq 12$ years of education were compared with norms established by the ALIVE study, ${ }^{5}$ whereas scores of subjects with $>12$ years of education were compared with norms established by the Multicenter AIDS Cohort Study. ${ }^{6}$ Cognitive impairment was defined as performance that was 2 SDs below the appropriate mean on one test or $1 \mathrm{SD}$ below the mean on two tests.

The eight tests included in the core neuropsychological battery covered six domains. Verbal memory was assessed with the Rey Auditory Verbal Learning Test. ${ }^{7}$ Impaired performance on any of the components of the test (Rey total score, fifth trial score, delayed recall, interference, or correct recognition) was sufficient to meet criteria for verbal memory impairment. Visual memory was assessed with the Rey Complex Figure Recall test. ${ }^{7}$ Constructional skills were assessed with the Rey Complex Figure Copy test. $^{7}$ Psychomotor skills were measured with the Digit Symbol test. ${ }^{*}$ Motor skills were assessed with the grooved pegboard (either dominant or nondominant hand) ${ }^{9}$ and timed-gait tests. If timed-gait performance was the only measure in which subjects scored 2 SDs below the mean, subjects were not considered to have met criteria for cognitive impairment. Frontal systems were assessed with the verbal fluency ${ }^{10}$ and the odd-man-out tests. ${ }^{11}$

Two other tests were administered but were not included in the neuropsychological core battery used in the AAN algorithm to determine the degree of cognitive impairment. The California Computerized Assessment Package, ${ }^{12}$ a measure of reaction time, was performed at $\mathrm{Co}$ lumbia and Johns Hopkins but not at Rochester. The National Adult Reading Test ${ }^{13.14}$ was administered to all subjects.

Functional assessment. Functional measures were chosen that would reflect the degree to which cognitive deficits compromised everyday function, a requirement for the diagnoses of dementia and cognitive impairment by the AAN Task Force. As stated in the task force report, "The major difference between HIV-1-associated dementia complex and HIV-1-associated minor cognitive/motor disorder is the degree of impairment in the activities of daily living. Because there are no widely accepted methods for determining a patient's degree of impairment in work, social or other daily activities, determining the degree of impairment caused by cognitive dysfunction may be based on clinical judgement." 2 Rather than relying on clinical judgment, we included standardized measures that reflected functional capabilities enumerated by the task force report for both HIV-1-associated cognitive/motor complex (i.e., $\mathrm{ADC}$ ) and minor cognitive/motor disorder. Measures were derived from the Instrumental Activities of Daily Living (IADL) scales of Lawton and Brody, ${ }^{15}$ the Katz Activities of Daily Living (ADL)/Lawton Personal Self-Maintenance scale, ${ }^{16}$ and the Role Functioning items of the Medical Outcomes Study (MOS). ${ }^{17}$ To meet the functional deficit criterion for dementia, respondents were required to report deficits in at least one of the eight IADL scales. We required subjects to determine whether IADL, ADL, and role functioning deficits could be attributed to a cognitive source, however, such attributions were used for staging subjects for severity of dementia. Information on work sta- tus (including volunteer work) and weekly hours of work was also collected.

Two functional outcomes were included that stress physical strength and stamina. The Karnofsky Performance Scale ${ }^{1 s}$ ranges from 0 to $100 \%$. A score of $<80 \%$ suggests the need for assistance with some ADL and an inability to work outside the home. The MOS Physical Function Subscale ${ }^{17}$ is a self-rated scale of nine activities. Each physical functioning item is rated from 1 (greatly limited) to 3 (not limited), yielding a maximum score of 27 (no physical limitations). This scale probes whether subjects can perform vigorous or moderate physical activity and whether they are limited in grocery shopping, climbing several flights of stairs or one flight, bending, walking more than one mile, walking more than one block, or walking one block. Neither the Karnofsky scale nor the MOS Physical Function Scale was part of the algorithm for determining ADC.

Psychiatric assessment. The 20-item Center for Epidemiologic Studies-Depression Scale (CES-D) ${ }^{19}$ was used to assess mood. If subjects' responses were indicative of a major affective disorder with severe depression, they were offered a formal psychiatric evaluation and were not included in the cohort.

Laboratory assessment. CD4 lymphocyte counts, hemoglobin, hematocrit, and beta $\mathrm{a}_{2}$ microglobulin levels were obtained.

Operationalization of the AAN criteria for $A D C$ and HIV-1-associated minor cognitive/motor disorder. The extensive functional evaluation and standardized neurologic, neuropsychological, and psychiatric assessments were used to develop an algorithm with which to classify subjects as either not meeting AAN criteria for significant cognitive impairment or impaired with either HIVassociated minor cognitive/motor disorder or ADC. Table 1 presents the algorithm in detail. To receive a diagnosis of ADC, subjects had to fulfill both cognitive and functional criteria, in addition to either neurologic or neuropsychiatric criteria. Demented subjects were further classified into three groups: mild, moderate, and severe. Subjects who did not meet criteria for dementia but had at least two deficits in cognitive tests or neurologic examination (Digit Symbol, Rey auditory verbal memory, finger agility, alternating movement, gait and coordination, limb coordination, emotional lability) and a deficit in at least one role function measure attributed to a cognitive source were classified as having an HIV-1-associated minor cognitive/motor disorder.

Data analysis. Demographic, laboratory, and clinical characteristics of subjects were compared among the three participating sites using analysis of variance (for comparing means) and chi-square tests (for comparing proportions). Multiple comparisons among sites were performed using Tukey's studentized range procedure (for means) and the Bonferroni correction (for proportions). The Kruskal-Wallis test was used to compare groups for variables not satisfying the assumption of normality.

Similar methods were used to compare demographic, laboratory, clinical, neurologic, neuropsychological, and behavioral characteristics of subjects among the AAN classification groups determined with the algorithm.

Multiple regression analysis was used to identify potentially important predictors of global functional status. Two outcomes were analyzed: the Karnofsky Performance Scale 
Table 1 Modification of AAN algorithm

\section{HIV-1-associated dementia complex (ADC)}

Criteria for 1 and 2 must be met:

1. Scores 1 SD below age- and education-adjusted norms on two of eight neuropsychological tests or 2 SDs below the norms on one of eight tests

2. Requires assistance or has difficulty (due to either physical or cognitive deficit) in one of the following IADL:

Using the telephone

Handling money

Taking medication

Performing light housekeeping

Doing laundry

Preparing meals

Shopping for groceries

Getting to places out of walking distance

and

Must meet either 1 or 2 of the following:

1. Any impairment in the following: lower extremity strength, coordination, finger tapping, alternating hand movements, leg agility, or performance on grooved pegboard 2 SDs below mean (dominant hand)

2. Self-reported frequent depression that interferes with function, loss of interest in usual activities or emotional lability, or irritability

Staging of $A D C$

Mild: must attribute IADL to a cognitive source

Moderate: satisfies neurologic and psychiatric criteria for mild $\mathrm{ADC}$ plus functional impairment in either telephone use, medication taking, or money handling plus two or three other IADLs or ADLs (indoor mobility, eating, dressing, grooming, toileting, getting in and out of bed, bathing) attributed to a cognitive source

Severe: satisfies neurologic and psychiatric criteria for moderate $\mathrm{ADC}$ and has in addition to impairment in telephone use, medication taking, or money handling at least four IADLs or ADLs attributed to a cognitive source

\section{HIV-1-associated minor cognitive/motor disorder}

Does not meet criteria for HIV-1-associated cognitive/motor disorder and meets 1 and 2 of the following:

1. Deficit in at least two of the following:

Mental slowing: digit symbol at least 1 SD below age- and education-adjusted norms

Memory: Rey Auditory Verbal learning test (total) at least 1 $\mathrm{SD}$ below norms

Motor dysfunction: any impairment in finger tapping or pronation/supination

Incoordination: mild impairment in gait or clumsiness

Emotional lability or apathy/withdrawal

and

2. Deficit in at least one of the role function measures attributed in part to cognitive function:

Need for frequent rests

Cut down on amount of time in activities

Accomplish less than desired

Cannot perform activities as carefully as one would like

Limited in work or activities

Difficulty performing activities

Requires special assistance to perform activities and the MOS Physical Function Subscale. The predictors considered for inclusion in the models were gender, years of education, CES-D score, hemoglobin level, square root of the number of medications, number of HIV diagnoses (less than or equal to one, or more than one), AAN classification group (nonimpaired, minor cognitive/motor disorder, or mild dementia), age, and log (CD4 count).

Results. Recruitment and composition of the cohort. Recruitment of the cohort at each site began in February 1994 and was concluded in December 1995. As of December $31,1995,271$ subjects had been recruited at the three sites. Each site used slightly different methods for recruitment. At Columbia University, subjects were recruited from a variety of sources, including a previous natural history study at the HIV Center for Clinical and Behavioral Studies, and targeted outreach (through newsletters and announcements) to the Gay Men's Health Crisis Center, People With AIDS Coalition, Housing Works, Inc., the Experimental Treatment Guide for New York State, day treatment centers, and other New York City AIDS centers. At The Johns Hopkins University, the majority of subjects were recruited from AIDS clinics at the hospital, but advertisements were also placed in newspapers and at community centers. At the University of Rochester, recruitment at the AIDS Clinic at Strong Memorial Hospital and from several AIDS clinics around the city were the primary sources of subjects.

At Columbia, 120 subjects were screened, and 95 were accepted into the natural history cohort. The 25 subjects who were not accepted into the study included 11 who did not meet the cognitive or CD4 count criteria, 6 who were depressed and referred for evaluation, and 4 who had primary untreated syphilis. Other reasons for nonparticipation included blindness, refusal to consider a drug trial if offered, stroke, severely abnormal liver function, primary language not English or Spanish, and lack of venous access. At Johns Hopkins, 116 subjects were screened, and 114 were entered into the cohort. At University of Rochester, all 62 subjects who were screened entered the cohort.

Baseline demographics by site. The mean log (CD4), percentage of participants with CD4 counts of $<200$, and mean hemoglobin for men and women did not significantly differ among the sites except for log beta $\mathrm{a}_{2}$ microglobulin, which was significantly lower $(p<0.05)$ at both Columbia $(1.13 \pm 0.35)$ and Johns Hopkins $(1.29 \pm 0.39)$ than at Rochester (1.42 \pm 0.48$)$. A higher percentage of subjects at Rochester $(39 \%)$ than at Columbia $(8 \%)$ or Johns Hopkins (29\%) reported Karnofsky scores of $\leq 70(p<0.001)$, indicating that a greater number of subjects were unable to work but were able to live at home with a varying amount of assistance. Although laboratory measures were similar among sites, Rochester subjects had significantly more extrapyramidal signs ( $p<0.001$ ), reported more difficulty with gait and coordination, and took significantly more medications than did subjects at the other two sites ( $p<$ 0.001 ). The percentage of subjects at each site who met criteria for dementia did not significantly differ (Columbia, $22.1 \%$; Johns Hopkins, 23.7\%; Rochester, $27.4 \%$ ), nor did the percentage of subjects who met criteria for minor cognitive/motor disorder differ significantly (Columbia, $24.2 \%$; Johns Hopkins, 21.1\%; Rochester, 16.1\%).

Despite the stipulated entry criteria, 46 of the subjects with a CD4 count of $>200$ did not meet criteria for cogni- 
Neuropsychological

Impairment

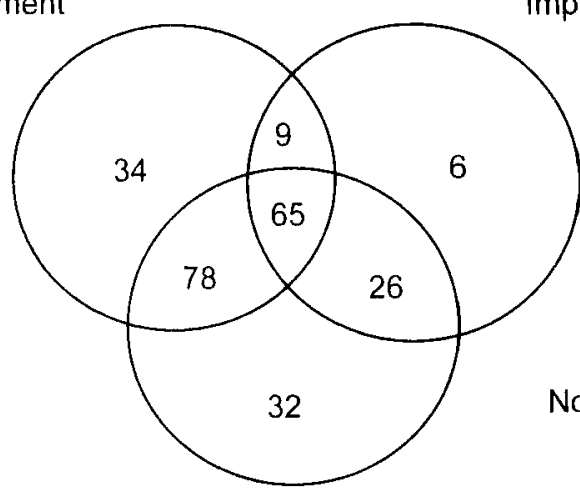

No Impairment 21

Neurologic/Behavioral Impairment

Figure. The Venn diagram shows which subjects in the cohort meet neuropsychological, functional, and neurologic/behavioral criteria stipulated by the American Academy of Neurology algorithm for HIV-1-associated cognitive motor disorder.

tive impairment. However, only 21 of these subjects failed to meet either the functional or neurologic criteria used for diagnosing HIV-1-associated dementia.

$A A N$ algorithm. Based on the algorithm, 150 subjects $(55.4 \%)$ did not meet criteria for minor cognitive/motor disorder or $\mathrm{ADC}$ and were considered nonimpaired, 56 (20.7\%) met criteria for HIV-1-associated minor cognitive/ motor disorder, and $65(24.0 \%)$ met criteria for HIV-1$\mathrm{ADC}$. Of the latter, three subjects were moderately demented. The rest met criteria for mild dementia.

Twenty of the 65 subjects who met neuropsychological, neurologic, or psychiatric criteria for dementia failed to attribute impairment in IADL or ADL to a cognitive deficit. For the majority of subjects, no independent corroboration of functional status was available. These 20 subjects were compared with the 45 subjects who did attribute functional deficit to cognitive impairment. There were no significant differences between these groups in demographic or laboratory assessment or neuropsychological or psychiatric variables. The two groups differed only in that subjects attributing functional deficit to a cognitive source had a significantly slower gait. Therefore, these 20 subjects were included in the mildly demented group.

To better characterize the cohort, we examined the distribution of subjects meeting neuropsychological, functional, and either neurologic or behavioral criteria. As seen in the figure, 65 people satisfied all three criteria, yielding the 65 subjects diagnosed with ADC. Seventy-eight subjects met both the neuropsychological and neurologic/ behavioral criteria but did not demonstrate any functional impairment. Only nine subjects met the neuropsychological and functional criteria without meeting the neurologic/ behavioral criteria, and six met the functional criteria alone. This algorithm suggests that it is very unusual for persons who meet the inclusion and exclusion criteria for this study to have both neuropsychological and functional impairments without some neurologic or behavioral disorder. Twenty-one patients did not meet any of the criteria for impairment in the three domains.

Demographic and laboratory assessments of the sub-
Table 2 Comparison of subjects not meeting criteria for minor cognitive/motor disorder and those meeting criteria for minor cognitive/motor disorder, and ADC on demographic, laboratory, and clinical measures

\begin{tabular}{|c|c|c|c|}
\hline Measure & $\begin{array}{c}\text { Nonimpaired } \\
(\mathrm{n}=150)\end{array}$ & $\begin{array}{c}\text { Minor } \\
\text { cognitive/ } \\
\text { motor } \\
\text { disorder } \\
(n=56)\end{array}$ & $\begin{array}{c}\mathrm{ADC} \\
(\mathrm{n}=65)\end{array}$ \\
\hline \multicolumn{4}{|l|}{ Demographic } \\
\hline Age (yr) & $40.6 \pm 7.7 \uparrow$ & $39.4 \pm 6.5$ & $37.9 \pm 7.8$ \\
\hline Men $(\%)$ & 78.7 & 82.1 & 73.9 \\
\hline Education (yr) & $13.7 \pm 2.9$ & $13.4 \pm 2.8$ & $13.3 \pm 3.2$ \\
\hline White $(\%)$ & 52.7 & 46.4 & 49.2 \\
\hline Black $(\%)$ & 40.0 & 42.9 & 35.4 \\
\hline Hispanic (\%) & 4.0 & 8.9 & 13.3 \\
\hline $\begin{array}{l}\text { Work/volunteer } \\
40 \mathrm{hr}(\%)\end{array}$ & 18.6 & 9.1 & $4.9^{*}$ \\
\hline \multicolumn{4}{|l|}{ Laboratory } \\
\hline CD4 $<200(\%)$ & 63.4 & 47.2 & 67.8 \\
\hline Hemoglobin & $12.9 \pm 1.8$ & $13.4 \pm 1.4^{\dagger}$ & $12.1 \pm 2.1 \dagger$ \\
\hline $\begin{array}{l}\log _{\text {beta }} \\
\text { microglobulin }\end{array}$ & $1.2 \pm 0.4$ & $1.2 \pm 0.4$ & $1.3 \pm 0.5$ \\
\hline \multicolumn{4}{|l|}{ Medical status } \\
\hline No. of medications & $5.3 \pm 3.5$ & $5.4 \pm 4.0$ & $7.3 \pm 6.1$ \\
\hline $\begin{array}{l}>1 \text { HIV diagnosis } \\
(\%)\end{array}$ & 11.3 & 10.7 & $15.4^{\mathrm{k}}$ \\
\hline \multicolumn{4}{|l|}{ Functional outcome } \\
\hline Karnofsky $<80 \quad(\%)$ & 16.9 & 16.1 & $43.8^{*}$ \\
\hline $\begin{array}{l}\text { MOS Physical } \\
\text { Function Subscale } \\
\text { score }\end{array}$ & $22.4 \pm 4.1$ & $20.1 \pm 4.3$ & $17.4 \pm 5.0^{*}$ \\
\hline \multicolumn{4}{|l|}{ Neurologic $(\%)$} \\
\hline Without EPS (\%) & 47.5 & 40.4 & $23.6^{*}$ \\
\hline $\begin{array}{l}\text { UPDRS Motor } \\
\text { Subscale score }\end{array}$ & $3.2 \pm 5.6$ & $3.7 \pm 6.5$ & $7.3 \pm 6.1^{*}$ \\
\hline \multicolumn{4}{|l|}{ Behavioral } \\
\hline CES-D & $16.9 \pm 10.9 \dagger$ & $23.2 \pm 9.3 \dagger$ & $25.5 \pm 10.7$ \\
\hline
\end{tabular}

Values are in percent or mean \pm SD.

$* p<0.05$.

$\dagger$ Significant difference.

ADC $=$ associated dementia complex; HIV $=$ human immunodeficiency virus; EPS $=$ extrapyramidal signs; $\operatorname{MOS}=$ Medical Outcomes Study; UPDRS = Unified Parkinson's Disease Rating Scale; CES-D = Center for Epidemiological Studies-Depression Scale.

jects who did not meet AAN criteria for impairment, of those who met criteria for minor cognitive impairment, and of those who were demented are presented in table 2. Subjects who did not meet criteria for impairment were significantly older on average than were those with ADC. Gender, education, and HIV risk group did not significantly differ among these groups. The mean log (CD4 count) differed among the groups, but the percentage of subjects with CD4<200 did not. Mean hemoglobin and 
Table 3 Comparison of neuropsychological, neurologic, and psychiatric profiles among subjects who do not meet AAN criteria for cognitive impairment, those who meet $A A N$ criteria for minor cognitive/motor disorder, and those who meet AAN criteria for $A D C$

\begin{tabular}{|c|c|c|c|}
\hline Criteria & $\begin{array}{c}\text { No cognitive } \\
\text { impairment } \\
(n=150)\end{array}$ & $\begin{array}{c}\text { Minor } \\
\text { cognitive/ } \\
\text { motor } \\
\text { disorder } \\
(\mathrm{n}=56)\end{array}$ & $\begin{array}{c}\mathrm{ADC} \\
(\mathrm{n}=65)\end{array}$ \\
\hline $\begin{array}{l}\text { Neuropsychological criteria } \\
\text { met }(\%)^{*: *}\end{array}$ & 58.0 & 60.7 & $100.0 \dagger$ \\
\hline Rey Auditory Verbal & 18.7 & 26.8 & $43.1 \dagger$ \\
\hline Rey Complex Figure Copy & 20.7 & 17.9 & 30.8 \\
\hline $\begin{array}{l}\text { Rey Complex Figure } \\
\text { Recall }\end{array}$ & 7.3 & 7.1 & 9.2 \\
\hline Grooved pegboard & 17.3 & 25.0 & $47.7 \dagger$ \\
\hline Symbol digit & 8.0 & 12.5 & 15.4 \\
\hline Odd-man-out & 9.3 & 10.7 & 18.5 \\
\hline Verbal fluency & 2.7 & 5.4 & 4.6 \\
\hline Timed-gait & 19.3 & 25.0 & $40.0 \dagger$ \\
\hline Neurologic criteria met $(\%)$ & 38.7 & 57.1 & $87.7 \dagger$ \\
\hline Impaired strength (legs) & 6.4 & 16.0 & $25.4 \dagger$ \\
\hline Impaired coordination & 26.2 & 32.0 & $65.1 \uparrow$ \\
\hline Impaired finger taps & 24.2 & 36.0 & $64.1 \dagger$ \\
\hline Impaired leg agility & 22.1 & 20.8 & $49.2 \dagger$ \\
\hline Psychiatric criteria met (\%) & 39.3 & 75.0 & $83.1 \dagger$ \\
\hline Depressed mood & 6.5 & 24.0 & $39.7 \dagger$ \\
\hline Apathy & 28.2 & 42.0 & $64.1 \dagger$ \\
\hline Emotional lability & 29.3 & 64.3 & 56.9 \\
\hline
\end{tabular}

* 2 SDs below mean.

$\dagger p<0.01$.

$\mathrm{ADC}=$ AIDS dementia complex. hematocrit were significantly lower in the demented group than in the cognitively impaired and unimpaired groups. Demented subjects were significantly more likely to have a Karnofsky score of $<80$. On neurologic examination, the three groups differed significantly in total motor UPDRS score.

Specific deficits within each of the defined groups. Nonimpaired, minor cognitive/motor deficit, and demented groups were compared to explore which deficits in neuropsychological, neurologic, and psychiatric function were prominent in impaired subjects (table 3 ). Neuropsychological performance 2 SDs below the mean in memory and speed of processing was significantly different among groups. The groups also differed significantly in all neurologic signs and self-reported behavioral measures. These behavioral attributes were elicited by the neurologist and were not part of the CES-D.

Multivariate analysis. To assess whether dementia status independently predicted functional outcomes, we regressed Karnofsky scores on a series of sociodemographic (age, education, gender), disease severity (log CD4, hemoglobin, square root of the number of medications, HIVrelated diagnoses dichotomized $\leq 1,>1$ ), and psychiatric (depression) indicators (table 4 ). We repeated the multivariate analyses with physical function score as the outcome (see table 4). In both cases, dementia status independently predicted poorer functional status. For the Karnofsky Performance Scale, mild dementia predicted poorer function, after adjusting for other model predictors, but minor cognitive/motor disorder was not independently associated with poorer function. With physical function score as an outcome, both minor cognitive/motor disorder and $\mathrm{ADC}$ were associated with significantly poorer function. CD4 count was not a significant predictor in the models.

Discussion. In this cohort of 271 subjects at high risk for the development of HIV dementia and related cognitive disorders, we demonstrated that it is

Table 4 Multiple regression analyses for prediction of functional outcomes

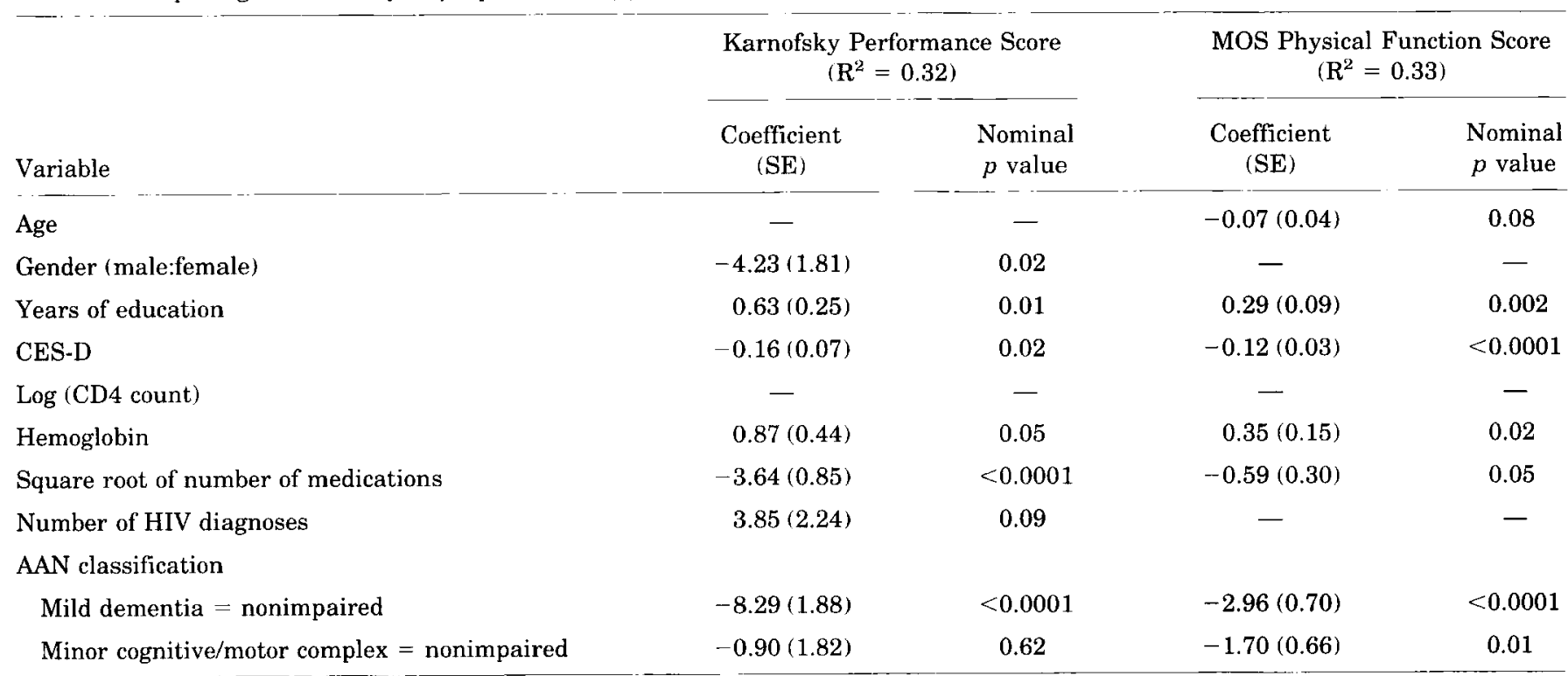

CES-D = Center for Epidemiological Studies-Depression Scale; HIV = human immunodeficiency virus. 
possible to operationalize the AAN definitions for $\mathrm{ADC}$ and minor cognitive/motor disorder using standardized functional, neurologic, and neuropsychological measures. We showed that it is rare to meet both the neuropsychological and functional criteria without meeting the neurologic criteria and that it is equally rare to meet functional criteria in isolation. The groups defined by the algorithm differed in log (CD4) count and mean hemoglobin. A number of neuropsychological tests of memory, attention, and speeded processing significantly differed among the groups.

We and others have previously demonstrated that these neuropsychological domains are preferentially affected with disease progression. In both gay men ${ }^{20}$ and male and female intravenous drug users, ${ }^{21,22}$ more severe HIV infection was associated with impairment in memory and executive function and reduced learning effects for memory motor speed and language tasks. The only neurologic test that distinguished the groups was the number of extrapyramidal signs. This may reflect the subcortical, primarily basal ganglia, involvement seen so commonly in ADC. In both gay men and intravenous drug users, CD4 count decline was associated with the development of extrapyramidal signs, impairment in rapid alternating movement, and frontal release signs..$^{23,24}$ Quantitative MRI studies have shown that basal ganglia structures show a selective atrophy in ADC. ${ }^{25}$ In autopsy studies of 21 of 55 brains of HIV1-infected patients, Brew et al. ${ }^{26}$ measured p-24 antigenemia and demonstrated that the globus pallidus contained the highest levels of immunostaining. The predominance of infection in the diencephalon and white matter has also been noted in previous histopathologic studies. ${ }^{27,28}$ Results of our study also support previous work suggesting that hemoglobin, previously a marker for systemic disease progression, can be used as a predictor of ADC because there was a significant difference among groups. ${ }^{1}$

We have previously shown that there is a relationship between the presence of neuropsychological impairment and work disability, ${ }^{29}$ as well as modification of time use in HIV-infected men with neurologic and/or neuropsychological deficits. ${ }^{30} \mathrm{HIV}$-positive men who developed neuropsychological impairment were more than five times as likely to work $<20$ hours per week than were HIV-negative, cognitively normal men, even after controlling for baseline neurologic status, neurologic symptoms, and CD4 count. ${ }^{29}$ Both overall neurologic disability and extrapyramidal function were significantly associated with the percentage of activities at home, time spent at work, and the percentage of time under medical care. ${ }^{30}$

Although the relationship between the presence of AIDS and functional decline as measured with the MOS and other scales has been demonstrated previously, ${ }^{31-33}$ the importance of dementia status as a predictor of function independent of other systemic indicators of infection has not been emphasized. The multivariate analysis showed that dementia status was an independent predictor of both physical function score and the Karnofsky Performance Score, emphasizing the importance of cognitive impairment. This last finding is important because it shows that distinct indicators of functional ability (i.e., indicators not used in the algorithm for assigning subjects to dementia class) are predicted by severity of cognitive impairment, even after controlling for other sources of functional deficit. Indeed, in this model, even subjects with the minor cognitive/motor disorder showed significantly poorer physical function than did the nonimpaired group. The Karnofsky Performance Scale was less sensitive to differences between subjects with the minor complex and nonimpaired subjects.

The main conclusion from this research is that subjects with minor cognitive/motor disorder can be operationally distinguished from subjects with $\mathrm{ADC}$ and from nonimpaired subjects (by operationalization of AAN criteria). An open question is whether the minor cognitive/motor complex disorder represents the earliest phase of the process of dementia or a separate syndrome. Longitudinal data are required to better address this question definitively.

Appendix. The Dana Consortium on Therapy for HIV Dementia and Related Cognitive Disorders Comprises the following: Karen Marder, $M D, M P H$; Steven Albert, PhD; George Dooneief, MD, MPH; Yaakov Stern, PhD; Gayatri Ramachandran, MD; George Todak, MSW; Ronda Friedman-Clouse, RN, Carmen Polanco; Thomas Winston; Peter Schofield, MD, FRCP; and Richard Mayeux, MD, MSE, Columbia University (New York, NY); Gerald Dal Pan, MD, MPH; Ola Selnes, PhD; Ned Sacktor, MD; Deborah Hasenauer, MA; Deneen Esposito; Letitia NanceSproson, MHA; and Justin McArthur, MB BS, MPH, The Johns Hopkins University (Baltimore, $M D$ ); and Karl Kieburtz, MD, MPH; Giovanni Schifitto, MD; Michael McDermott, PhD; Donna Palumbo, PhD; Connie Orme; Carol Zimmerman, RN; Harris Gelbard, MD, PhD; Charlyne Hickey, RN; Cindy Casaceli; Barry Guthrie; Lisa Rumfola; and Leon Epstein, MD, University of Rochester (Rochester, NY).

\section{References}

1. McArthur JC, Hoover DR, Bacellar H, et al. Dementia in AIDS patients: incidence and risk factors. Neurology 1993;43: 2245-2252.

2. Janssen RS, Cornblath DR, Epstein LG, et al. Nomenclature and research case definitions for neurological manifestations of human immunodeficiency virus type-1 (HIV-1) infection: report of a Working Group of the American Academy of Neurology AIDS Task Force. Neurology 1991;41:778-785.

3. Fahn S, Marsden C, Calne D. Recent developments in Parkinson's disease. Florham Park, NJ: Macmillan Healthcare Information, 1987:153.

4. Butters N, Grant I, Haxby J, et al. Assessment of AIDsrelated cognitive changes: recommendations of the NIMH Workgroup on Neuropsychological Assessment Approaches. J Clin Exp Neuropsych 1990;12:963-978.

5. Concha M, Selnes OA, McArthur J, et al. Normative data for a brief neuropsychologic test battery in a cohort of injecting drug users. Intl J Addictions 1995;30:823-841. 
6. Selnes OA, Jacobson L, Machado AM, et al. Normative data for a brief neuropsychological screening battery. Percept Mot Skills 1991;73:539-550.

7. Rey A. L'examen psychologique dans les cas d'encephalopathie traumatique. Arch Psychol 1941;28:286-340.

8. Wechsler D. Wechsler Adult Intelligence Scale-Revised. New York, NY: The Psychological Corporation, 1981.

9. Klove H. Clinical neuropsychology. Med Clin North Am 1963; 46:1647-1658.

10. Benton AL. The Visual Retention Test. New York, NY: The Psychological Corporation, 1955.

11. Flowers KA, Robertson C. The effects of Parkinson's disease on the ability to maintain a mental set. J Neurol Neurosurg Psychiatry 1985;48:517-529.

12. Miller EN, Satz P, Visscher B. Computerized and conventional neuropsychological assessment of HIV-1 infected homosexual men. Neurology 1991;41:1608-1616.

13. Nelson HE. The National Adult Reading Test (NART): test manual. London: The National Hospital for Nervous Diseases, 1982.

14. Nelson HE, O'Connell A. Dementia: the estimation of premorbid intelligence levels using the National Adult Reading Test. Cortex 1978;14:234-244.

15. Lawton MP, Brody EM. Assessment of older people: selfmaintaining and instrumental activities of daily living. Gerontologist 1969;9:179-186.

16. Katz S, Ford A, Moskowitz R, et al. Studies of illness in the aged: the index of ADL. JAMA 1963;183:914-919.

17. Stewart AL, Ware JE. Measuring function and well-being: the Medical Outcomes Study approach. Durham, NC: Duke University Press, 1993.

18. Karnofsky DA, Abelman WH, Carver LF, et al. The use of nitrogen mustards in the palliative treatment of carcinoma. Cancer 1948;1:634-656.

19. Radloff LL. The CES-D: a self-report depression scale for research in the general population. Appl Psychol Meas 1977;1: $385-401$.

20. Stern Y, Liu X, Marder K, et al. Neuropsychological changes in a prospectively followed cohort of gay and bisexual men with and without HIV infection. Neurology 1995;45:467-472.

21. Stern Y, Liu X, Marder K, et al. Neuropsychological changes in a prospectively followed cohort of intravenous drug users with and without HIV. Neuropsychiat Neuropsychol Beh Neurol 1996;9:83-90.

22. Royal W, Updike M, Selnes OA, et al. HIV-1 infection and nervous system abnormalities among a cohort of intravenous drug users. Neurology 1992;42:1924-1930.

23. Marder K, Liu X, Stern Y, et al. Neurologic signs and symptoms in a cohort of homosexual men followed for 4.5 years. Neurology 1995;45:261-267.

24. Marder K, Liu X, Stern Y, et al. Risk of human immunodeficiency virus type 1-related neurologic disease in a cohort of intravenous drug users. Arch Neurol 1995;52:1174-1182.

25. Aylward EH, Henderer JD, McArthur JC, et al. Reduced basal ganglia volume in HIV-1-associated dementia: results from quantitative neuroimaging. Neurology 1993;43:2099-2104.

26. Brew BJ, Rosenblum M, Cronin K, Price R. AIDS dementia complex and HIV-1 brain infection: clinical-virological correlations. Ann Neurol 1995;38:563-571.

27. Navia B, Cho ES, Petito CK, Price RW. The AIDS dementia complex. II. Neuropathology. Ann Neurol 1986;19:525-535.

28. Kure K, Weidenheim KM, Lyman WD, Dickson DW. Morphology and distribution of HIV-1 gp41-positive microglia in subacute AIDS encephalitis: pattern of involvement resembling a multisystem degeneration. Acta Neuropathol (Berlin) 1990;80: 393-400.

29. Albert SM, Marder K, Dooneief G, et al. Neuropsychologic impairment in early HIV infection: a risk factor for work disability. Arch Neurol 1995;52:525-530.

30. Albert SM, Todak G, Elkin E, Marder K, Dooneief G, Stern Y. Time allocation and disability in HIV infection: a correlational study. J Occup Sci 1995;1:21-30.

31. Lubeck DP, Fries JF. Changes in quality of life among persons with HIV infection. Qual Life Res 1992;1:359-366.

32. Wu AW, Rubin HR, Mathews WC, et al. A health status questionnaire using 30 items from the Medical Outcomes Study: preliminary validation in persons with early HIV infection. Med Care 1991;29:786-798.

33. O'Dell MW, Lubeck DP, O'Driscoll P, Matsuno S. Validity of the Karnofsky performance status in an HIV-infected sample. J Acquir Immune Defic Syndr Hum Retrovirol 1995;10:350357. 